\title{
Effect of Electronic Medical Record Utilization on Depression, Anxiety and Stress among Doctors and Nurses in Johor, Malaysia
}

\author{
Ahmad Fairuz Mohamed ${ }^{1}$, Mohd Nazri Shafei ${ }^{2}$ and Mohd Ismail Ibrahim ${ }^{2}$ \\ 1. Department of Community Medicine, Faculty of Medicine, Cyberjaya University College of Medical Sciences (CUCMS), \\ Cyberjaya, Selangor 63000, Malaysia \\ 2. Department of Community Medicine, School of Medical Sciences, Universiti Sains Malaysia, Kubang Kerian, Kota Bharu, \\ Kelantan 16150, Malaysia
}

Received: April 16, 2015 / Accepted: April 30, 2015 / Published: April 30, 2015.

\begin{abstract}
Background: The usage of modern technology in healthcare record system is now a must throughout the world. However, many doctors and nurses has been reporting facing numerous challenges and obstacles in the implementation. The aim of the present study is to determine the prevalence of depression, anxiety and stress among doctors and nurses who utilize EMR (electronic medical record) and its associated factor. Methods: A comparative cross-sectional study was conducted from January till April 2012 among doctors and nurses in two public tertiary hospitals in Johor in which one of them uses EMR and the other one still using the MMR (manual medical record) system. Data was collected using self-administered validated Malay version of DASS-21 (Depression, Anxiety, and Stress Scales-21) items questionnaire. It comprises of socio-demographic and occupational characteristics. Findings: There were 130 respondents with a response rate of $91 \%$ for EMR and 123 respondents with a response rate of $86 \%$ for MMR. The mean (SD) age of respondents in EMR and MMR groups were 34.7 (9.42) and 29.7 (6.15) respectively. The mean (SD) duration of respondents using EMR was 46.1 (35.83) months. The prevalence of depression, anxiety and stress among respondents using EMR were $6.9 \%, 25.4 \%$ and $12.3 \%$. There were no significant difference between the study groups related to the depression, anxiety and stress scores. In multivariable analysis, the significant factors associated with depression among respondents using EMR was age (OR $1.10,95 \%$ CI 1.02, 1.19). The significant factors associated with stress among respondents using EMR was marital status (OR 3.33, 95\% CI 1.10, 10.09) and borderline significant was computer skill course (OR 2.94, 95\% CI 0.98, 8.78). Conclusion: The prevalence of depression, anxiety and stress of those who uses EMR were within acceptable range. Age, marital status and computer skill are the identified factor associated with the depression and stress level which need to be considered in its implementation.
\end{abstract}

Key words: Electronic medical record, depression, anxiety, stress, healthcare staff, associated factors.

\section{Background}

Healthcare system throughout the world is facing with sustainability issues as the need to meet high demands is disrupted with limited resources. Malaysia's Health Vision 2020 is an advanced and socially cohesive move to improve the standard of living and quality of life [1]. Hence, focusing on preventive approaches, supporting individual and family in making

Corresponding author: Ahmad Fairuz Mohamed, Md., research fields: occupational health, public health and telehealth.E-mail: fairuzila2003@gmail.com. lifestyle choices and providing services to support maintenance of health in a state of wellness are strategies employed to achieve sustainability of healthcare system with cost-effective. Therefore, the "Industrial Age Medicine" of healthcare pyramid needed to be inverted into "Information Age Healthcare" as showed in Fig. 1 [2]. Transformation here means that the financial and human resources should be shifted whereby instead of focusing at tertiary care institutions and curative services, focus should be given to preventive approaches at primary care level 


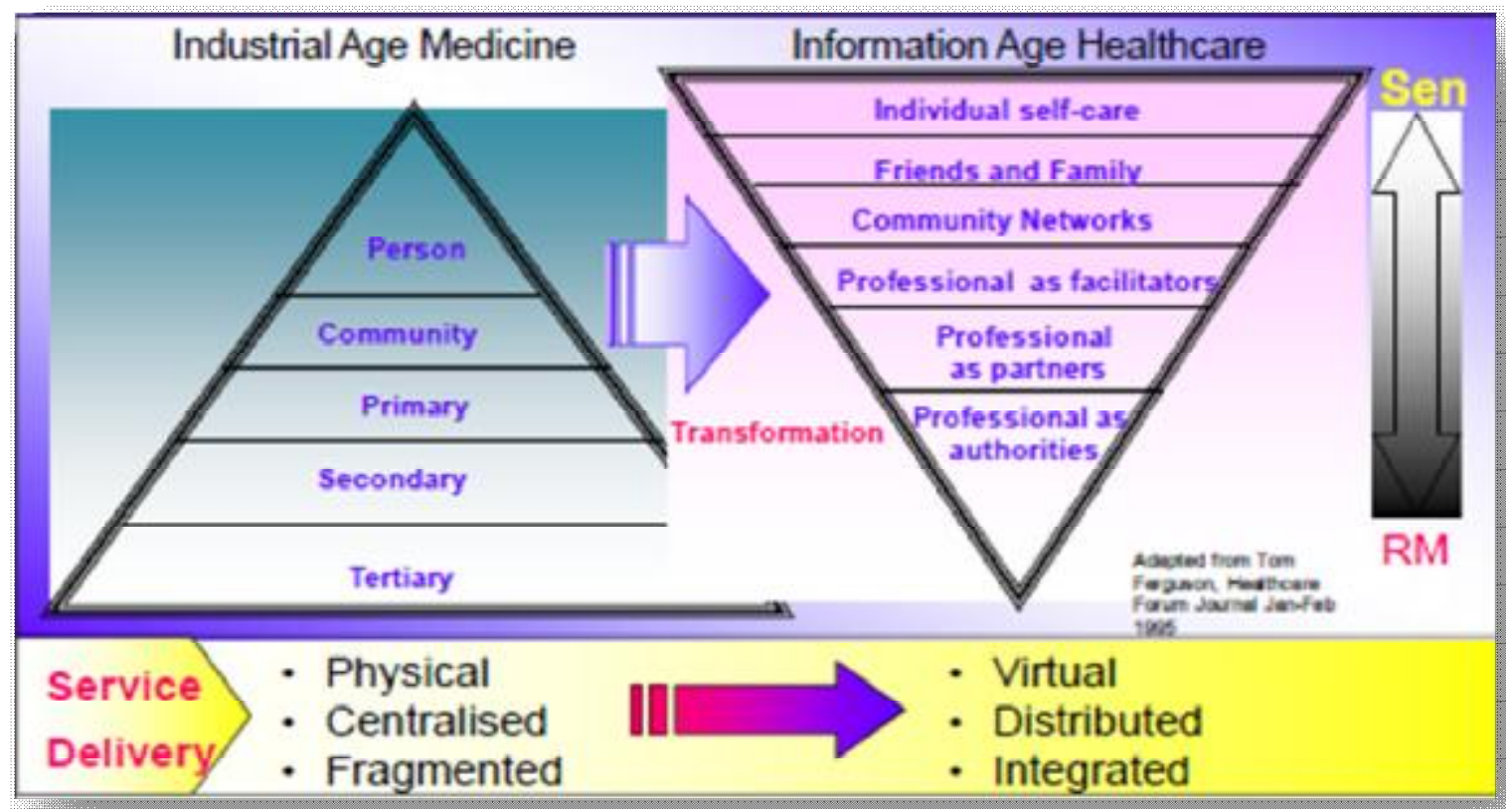

Fig. 1 Transforming industrial age healthcare system to information and communication age healthcare system.

and the services to be provided to individuals as close to home as possible.

The transformation towards future healthcare system will be supported and strengthened by telemedicine. Telemedicine is defined as the provision of healthcare and health-related services using telecommunications, information and multimedia technologies to link the participants in the healthcare system which are the healthcare providers, universities, public institutions, healthcare consumer, health care suppliers, and health care financing [1]. Telemedicine began with four pilot projects which are MCPHIE (Mass Customised Personalised Health Information and Education), CME (Continuing Medical Education), Teleconsultation and LHP (Lifetime Health Plan). The entire scope and their inter-relationships are as showed in Fig. 2. EMR (electronic medical record) is an electronic form of patient health information record digitally documented by healthcare providers in any healthcare settings [2]. EMR is a key element for telemedicine projects.

National study of clinical ICT (information communication and technologies) system that was conducted in Finland among physician showed that the EMR systems were very critical, did not support clinical works, physician needed to follow the fixed system, system did not support retrieving of data and physician suffered from non-integrated systems and system failures [3]. A study on the adoption of ICT among healthcare provider was conducted in UMMC (University Malaya Medical Centre). They found that among the problems related to ICT use were comments from healthcare providers; limited number of hardware provided; need to be shared, application system is always down; required longer time to perform job, old hardware; system always breakdown, internet was limited to get access and data is redundant [4]. Consequently, these problems will lead to stress build up among healthcare providers especially doctors and nurses.

Fig. 3 shows the model of work balance system of computer users and conceptual framework. Each component of job stressors has their own factors and among the components are interrelated and giving load to an individual. Personal capacity will cope and modify the load. If individuals are misfit to cope, it will lead to psychological, physiological and biomechanical strain. If the strains become chronic exposure to the individual, it will lead to health disorders. The psychological outcome will be manifested as depression, 


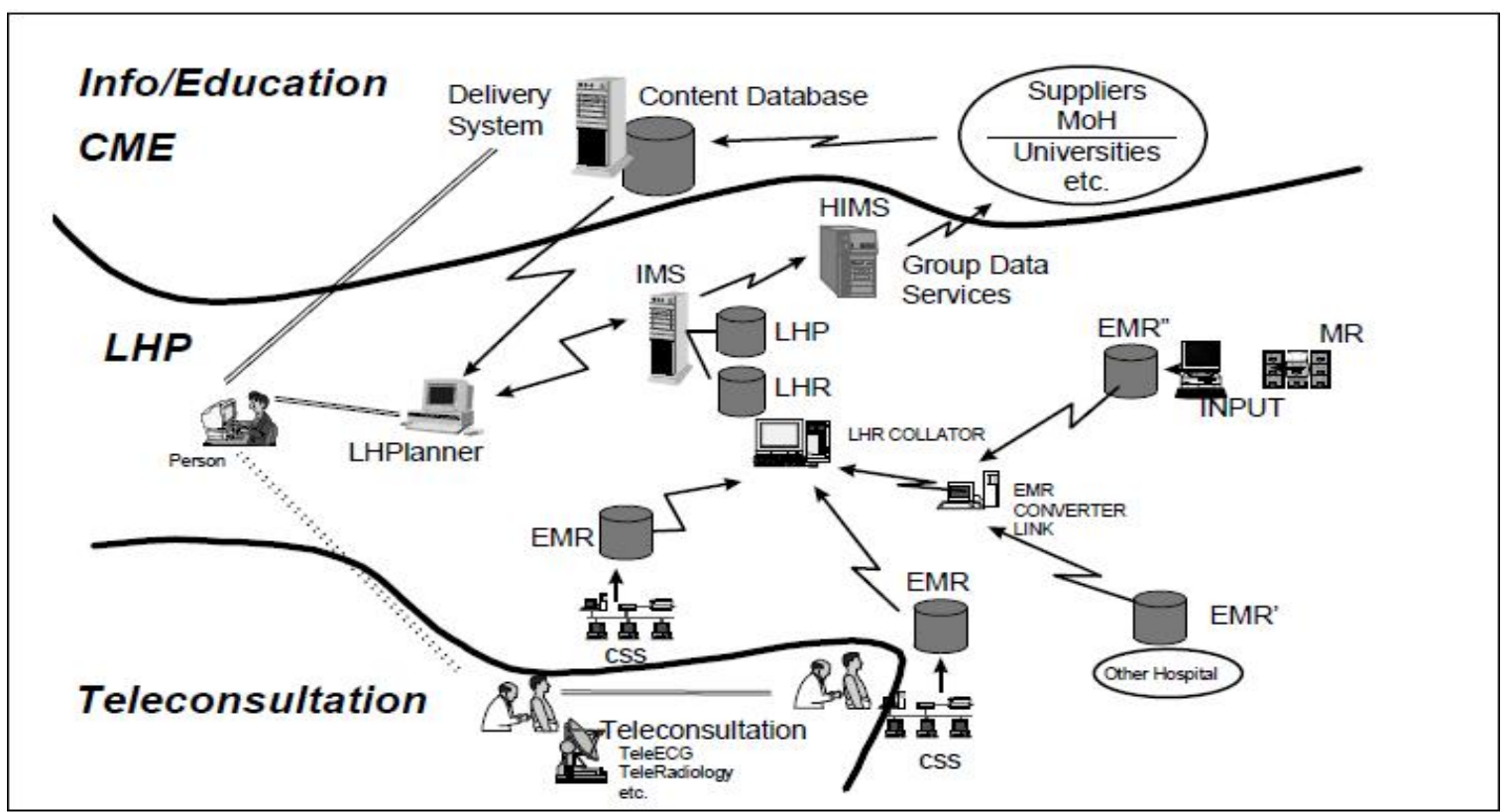

Fig. 2 Entire scope of telemedicine pilot projects.
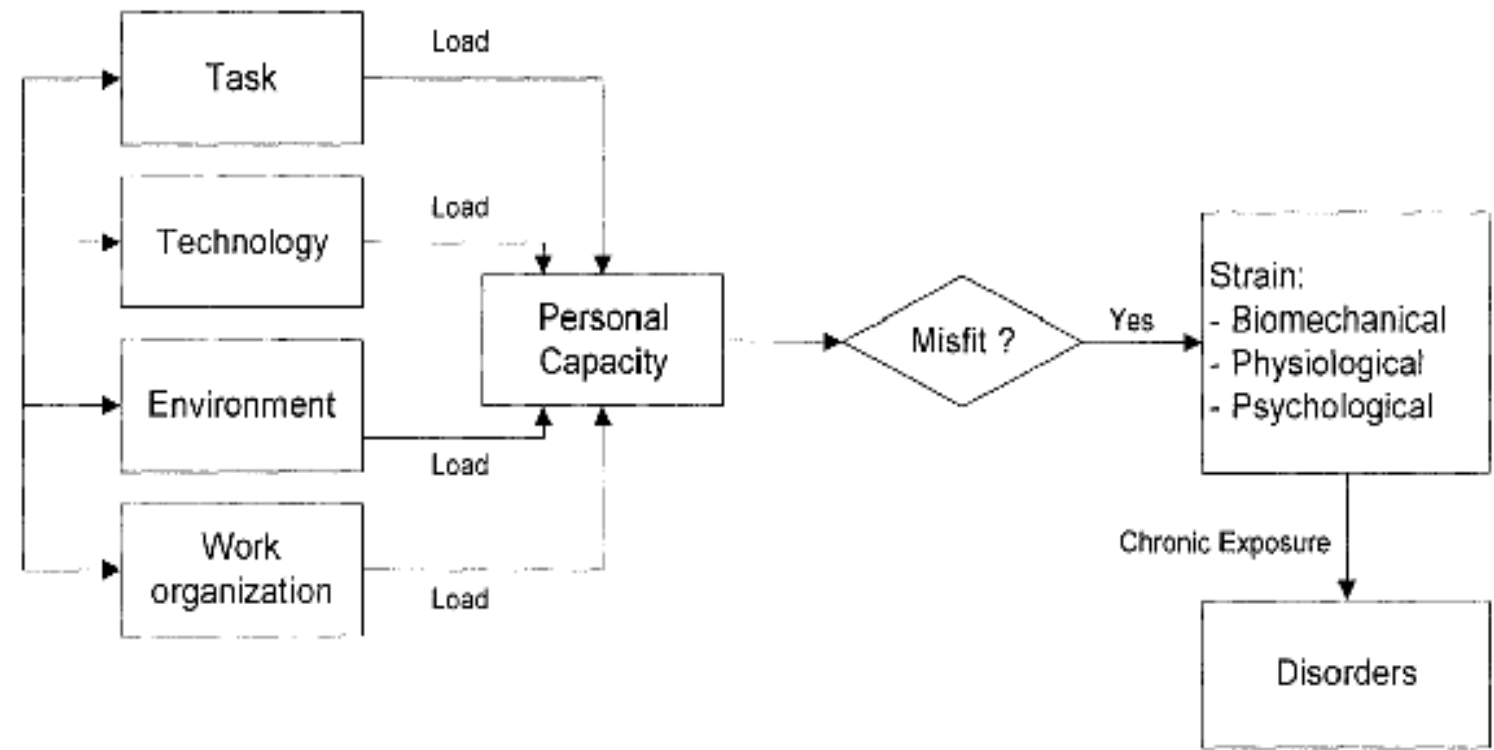

Fig. 3 Model of work system misfit.

anxiety and stress [5]. Therefore, the current study was conducted to provide the association of EMR usage towards mental health outcome and these will determine the efficiency of EMR systems and telemedicine projects.

\section{Methods}

\subsection{Study Design, Study Population and Setting}

This comparative cross-sectional study was conducted among all registered doctors and nurses working in public tertiary hospitals whereby one of them uses EMR and the other one uses MMR. Those aged between 21 to 58 years, and those who do not have any mental illness and have been using EMR for a period of at least 6 months represent the EMR group. Those aged between 21 to 58 years, not having any mental illness and never used EMR were included in the study to represent the MMR group. 


\subsection{Sampling Method and Data Collection Method}

The sample size was calculated by using single proportion formula and PS software [6] to compare two independent proportions. The minimum number of respondents required was 286 subjects. The proportionate stratified random sampling was applied to 286 subjects which was 143 subjects for each group. The strata identified was $70 \%$ of nurses and $30 \%$ of doctors.

Each respondent was required to answer a self-administered proforma and validated self-administered Malay version DASS (Depression, Anxiety and Stress Scale) 21 Items questionnaire [7].

\subsection{Statistical Analysis}

Data were entered and analyzed using SPSS (Statistical Program for Social Science) Version 19.0 [8]. The numerical data was presented as mean and standard deviation while the categorical was presented as frequency and percentage. Chi square test was used to determine the comparison between two categorical. Multiple logistic regressions were used to look for significant associated factors.

\subsection{Ethical Consideration}

The present study was approved by Research and Ethical Committee, School of Medicine Sciences, Universiti Sains Malaysia, Kelantan Health Campus on January 10, 2012, IHSR (Institute for Health System Research) on February 15, 2012 and MERC (Medical Ethics Research Committee) on February 23, 2012.

\section{Results}

\subsection{Socio-Demographic Characteristics}

A total of 123 respondents from MMR and 130 from EMR were eligible in the present study. There were no significant differences in sex, ethnic group, marital status, educational level and family psychiatric history related to depression and anxiety between EMR and MMR groups. It shows that the socio-demographic backgrounds between respondents from EMR and MMR group were homogenous.

\subsection{Occupational Characteristics}

The mean (SD) duration of respondents using EMR was 46.1 (35.83) months. There were no significant differences in term of nature of job, work pattern (shift work and working hours) and duration of working per day (hours) between MMR and EMR groups. It shows that occupational backgrounds of respondents were comparable between the two study groups. However, there were significant differences in position grade, duration of working and computer skill course between EMR and MMR groups. This is because the respondents from EMR group were provided with computer skills training.

\subsection{Prevalence of Depression, Anxiety and Stress}

The prevalence of depression, anxiety and stress among respondents from EMR group was as shown in Fig. 4. The proportion of depression, anxiety and stress were higher in EMR group compared to MMR group, but the differences were not statistically significant.

\subsection{Risk Factors of Depression, Anxiety and Stress}

Based on the authors' multivariate analysis on the factors identified associated with DAS, the study reported that one year increased in age of respondent, there will be 1.10 time the odds of them to have depression $(95 \%$ CI 1.02, 1.19, $p$-value $=0.017)$ after controlling for other confounders. Respondents who are single/widow/divorced have 3.33 time the odds to have stress compared to married respondents $(95 \% \mathrm{CI}$ $1.10,10.09, p$-value $=0.033)$ with the computer skill course factor adjusted. Respondents who did not have computer skill course have 2.94 times more in odds to have stress related problems compared to respondents who have trained computer skill. However the $p$-value was borderline $=0.054$ with $(95 \%$ CI $0.98,8.78)$ after adjusted for marital status factor. There is no factor significantly associated with the anxiety level. 


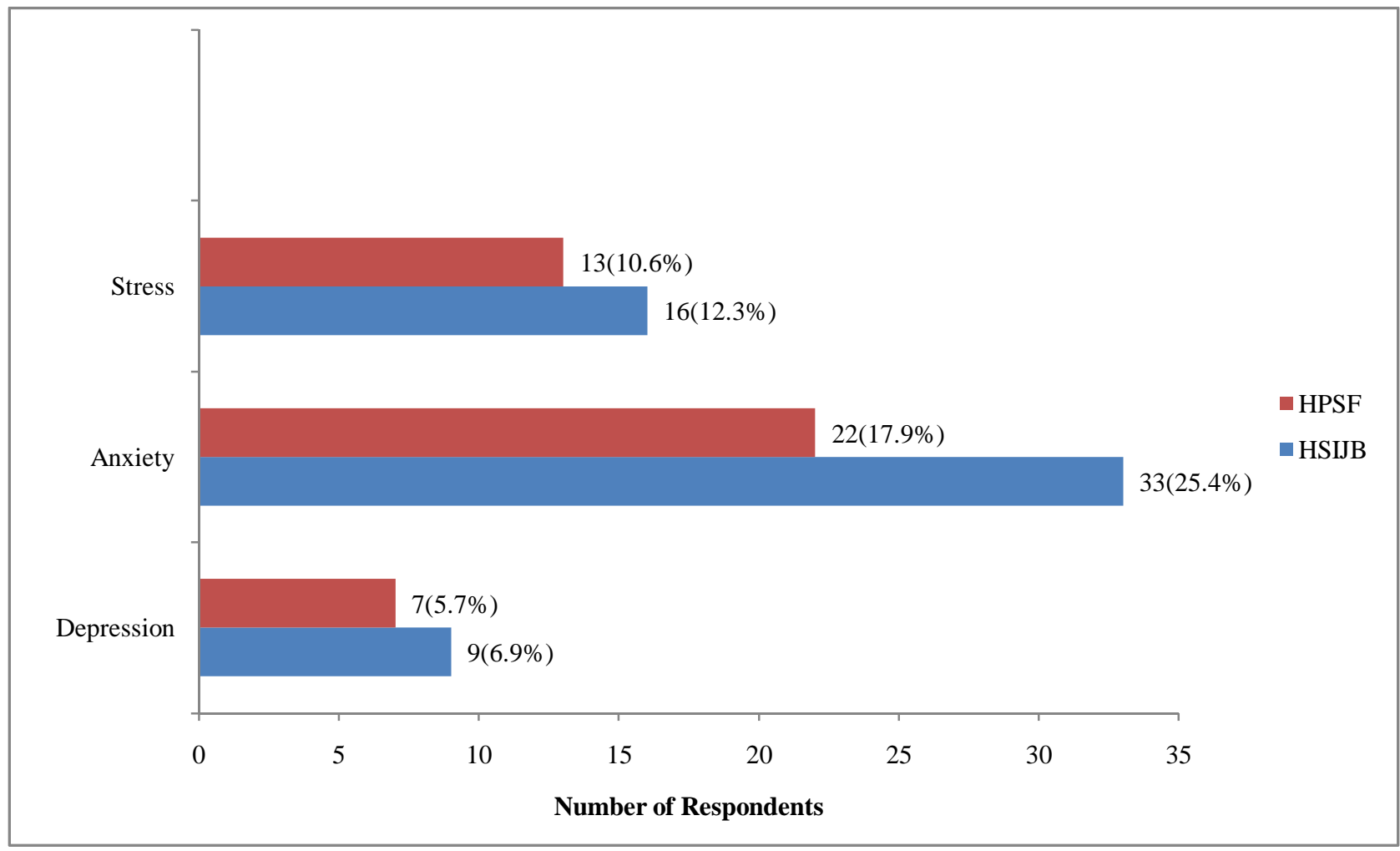

Fig. 4 Prevalence of depression, anxiety and stress among respondents.

\section{Discussions}

The proportion of DAS (depression, anxiety and stress) were higher in EMR group as compared to those in MMR group of representative. However, these differences were not statistically significant. The mean (SD) duration of doctors and nurses using EMR was 46.1 (35.8) months. The attendances rate for computer skill courses among doctors and nurses in EMR group was $70 \%$. This gives a reason why their DAS was relatively similar to those who have not been exposed to EMR. They have already adapted to the full computerization systems with the support of computer skill courses provided as part of the change management program. The finding was consistent with a study conducted by [9]. It was regarding clinician acceptance and performance study which post user training provided during 6 months EMR go live, then acceptance test post 6 months showed increased from $39 \%$ to $85 \%$ [9]. Under sample of respondents could be another potentially reason causing the similar finding.
Age is one of the risk factor of depression among respondents using EMR. Changes in a workplace affect individuals differently according to their age and stage in life. Working in computerization system with EMR will definitely change the work environment. This causes an individual to adapt to the stressor in order to prevent psychological strains in model of work system misfit [5]. Therefore age is an important predicted factor for respondents using EMR to have depression. An older age most probably have difficulties to adapt with new environment related to computerization systems whereby they need a skillful person who has up to date knowledge in the field of IT (information technology). Based on a study done in Denizli Hospital, the level of depression was higher among the older age strata compared to those who were younger [10].

Another significant associated factors of stress among respondents using EMR were ethnic group and marital status. There are many variables may affect individual vulnerability of occupational stress. The most important factors affecting stress response are 
personality structure, family life, stage in life and social support system. There are many cases of occupational stress which were influenced by factors in the personal sphere [11]. National Health Morbidity Survey 2006 shows that the psychiatric morbidity is higher among the divorces $(13.6 \%)$; followed by singles $(13.1 \%)$, widow/widower $(12.2 \%)$ and lastly those who were married (10.5\%). A study of psychosomatic symptoms of Japanese working women and their need for stress management show that the unmarried workers in the 30 to 44 age range complained as mental stress index (e.g. menstrual pain, diarrhea, neck/shoulder/arm pain) more frequently than married ones [12]. A cross-sectional study of occupational stress among 518 (90.4\% response rate) nurses in psychiatric institutions in Taiwan have 6.9 times the odds compared to married nurses to have occupational stress [13]. Both studies by Araki et al. [12] and Shen et al. [13] are consistent with current study findings. Therefore, the marital status was an important predictor of stress, what more if they were expose to high skill technology in their work like using EMR.

A paper review on occupational stress in human computer interaction states that computer tasks can produce physiological stress reactions such as changes in heart rate, blood pressure, catecholamine level, and brain wave activity. Computer users in less skilled jobs have greater amounts of stress compared to those in higher skilled jobs. When jobs are transitioned from one technology to a newer one, those employees who are less skilled report more stress due to newer technology than the employees who are skillful. A higher level of task difficulty coupled with inadequate skills is a job stressor that consistently become stressful across different categories [5]. Therefore, those doctors and nurses who work in a full computerization systems with inadequate computer skills will easily have stress as compared to those who have been trained.

\section{Conclusion}

The prevalence of depression, anxiety and stress respondents using EMR were within the range of prevalence depression, anxiety and stress in Malaysia and worldwide. There were no different in mental health outcome among those doctors and nurses using EMR compared to Non-EMR. This reflects the success of Telehealth projects in Malaysia by Ministry of Health and also reflects the good coping mechanism among the users. In the study, the authors found that the age was predicted factor for depression among respondents using EMR. In addition to this, computer skill course and marital status were also important factors that closely associate with the stress level among respondents using EMR.

\section{Acknowledgement}

The authors would like to extend their deepest gratitude to Department of Community Medicine, Universiti Sains Malaysia and Ministry of Health, Malaysia for granting permission to conduct this study. Special thanks to Director of Hospitals in Johor for giving me the cooperation in conducting this research project in their hospitals. They are most grateful to all respondents of this study which include the medical officers, head of nurses, matrons, sisters and other staff nurses who provide me with their valuable responses, assistance and cooperation in making this research a success.

\section{References}

[1] HIMSS. 2011. Electronic Health Record. Accessed November 14, 2011. http://www.himss.org/ASP /topics_ehr.asp.

[2] Amiruddin, H. 2010. Telehealth Malaysia. Accessed November 15, 2011. http://www.mscmalaysia.my/ codenavia/portals/msc/images/pdf/ihe_connectathon/Dr-2 0Amiruddin-20Hisan-20--20Telehealth-20Malaysia.pdf.

[3] Viitanen, J., Reponen, J., and Winblad, I. 2011. "National Questionnaire Study on Clinical ICT Systems Proofs: Physicians Suffer from Poor Usability." International Journal of Medical Informatics 80 (10): 708-25.

[4] Amnah, B., and Nik, R. 2010. "Adoption of ICT at Hospital A Case Study of UMMC." In Proceedings of the 2nd International Conference on Electronic Computer Technology (ICECT 2010). 


\section{Doctors and Nurses in Johor, Malaysia}

[5] Smith, M. J., Conway, F. T., and Karsh, B. T. 1999. "Occupational Stress in Human Computer Interaction." Ind Health 37 (2): 157-73.

[6] Dupont, W. D., and Plummer, W. D. 1990. "Power and Sample Size Calculations: A Review and Computer Program." Controlled Clinical Trials 11: 116-28.

[7] Ramli, M., Ariff, F. M., and Zaini, Z. 2007. "Translation, Validation and Psychometric Properties of Bahasa Malaysia Version of the Depression Anxiety and Stress Scales (DASS)." ASEAN Journal of Psychiatry 8 (2): 82-9.

[8] SPSS Inc. Released 2009. PASW Statistics for Windows, Version 18.0. Chicago: SPSS Inc.

[9] Krall, M. A. 1995. "Acceptance and Performance by Clinicians Using an Ambulatory Electronic Medical Record in an HMO." In Proceeding of the Annual
Symposium on Computer Applications in Medical Care, 708-11.

[10] Erdur, B., Ergin, A., Turkcuer, I., Parlak, I., Ergin, N., and Boz, B. 2006. "A Study of Depression and Anxiety among Doctors Working in Emergency Units in Denizli, Turkey." Emergency Medicine Journal 23 (10): 759-63.

[11] LaDou, J. 2007. Current Occupational \& Environmental Medicine, edited by Mc Graw, H. 846.

[12] Araki, Y., Muto, T., and Asakura, T. 1999. "Psychosomatic Symptoms of Japanese Working Women and Their Need for Stress Management." Industrial Health 37 (2): 253-62.

[13] Shen, H.-C., Cheng, Y., Tsai, P.-J., Lee, S.-h. S., and Guo, Y. L. 2005. "Occupational Stress in Nurses in Psychiatric Institutions in Taiwan." Journal of Occupational Health 47 (3): 218-25. 